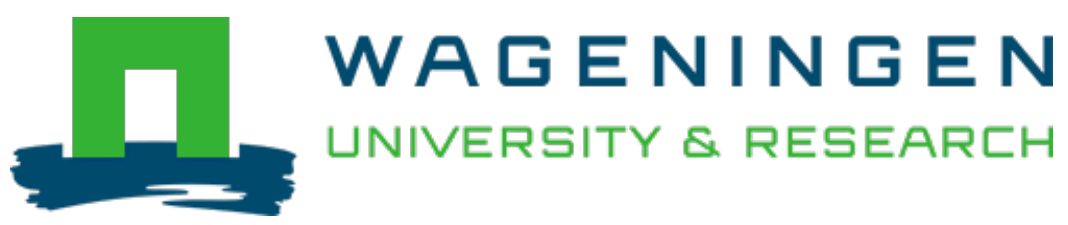

\title{
Microscopic insights into the failure of elastic double networks
}

Physical Review Materials

Tauber, Justin; Dussi, Simone; Gucht, Jasper

https://doi.org/10.1103/PhysRevMaterials.4.063603

This article is made publicly available in the institutional repository of Wageningen University and Research, under the terms of article $25 \mathrm{fa}$ of the Dutch Copyright Act, also known as the Amendment Taverne. This has been done with explicit consent by the author.

Article $25 \mathrm{fa}$ states that the author of a short scientific work funded either wholly or partially by Dutch public funds is entitled to make that work publicly available for no consideration following a reasonable period of time after the work was first published, provided that clear reference is made to the source of the first publication of the work.

This publication is distributed under The Association of Universities in the Netherlands (VSNU) 'Article $25 \mathrm{fa}$ implementation' project. In this project research outputs of researchers employed by Dutch Universities that comply with the legal requirements of Article $25 \mathrm{fa}$ of the Dutch Copyright Act are distributed online and free of cost or other barriers in institutional repositories. Research outputs are distributed six months after their first online publication in the original published version and with proper attribution to the source of the original publication.

You are permitted to download and use the publication for personal purposes. All rights remain with the author(s) and / or copyright owner(s) of this work. Any use of the publication or parts of it other than authorised under article $25 \mathrm{fa}$ of the Dutch Copyright act is prohibited. Wageningen University \& Research and the author(s) of this publication shall not be held responsible or liable for any damages resulting from your (re)use of this publication.

For questions regarding the public availability of this article please contact openscience.library@,wur.nl 


\title{
Microscopic insights into the failure of elastic double networks
}

\author{
Justin Tauber $\odot,{ }^{*}$ Simone Dussi $\odot,{ }^{\dagger}$ and Jasper van der Gucht ${ }^{\ddagger}$ \\ Physical Chemistry and Soft Matter, Wageningen University, Stippeneng 4, 6708 WE, Wageningen, The Netherlands
}

(Received 1 February 2020; revised manuscript received 17 April 2020; accepted 19 May 2020; published 8 June 2020)

\begin{abstract}
The toughness of a polymer material can increase significantly if two networks are combined into one material. This toughening effect is a consequence of a transition from a brittle to a ductile failure response. Although this transition and the accompanying toughening effect have been demonstrated in hydrogels first, the concept has been proven effective in elastomers and in macroscopic composites as well. This suggests that the transition is not caused by a specific molecular architecture, but rather by a general physical principle related to the mechanical interplay between two interpenetrating networks. Here we employ theory and computer simulations, inspired by this general principle, to investigate how disorder controls the brittle-to-ductile transition both at the macroscopic and the microscopic level. A random spring network model featuring two different spring types enables us to study the joined effect of initial disorder and network-induced stress heterogeneity on this transition. We reveal that a mechanical force balance gives a good description of the brittle-to-ductile transition. In addition, the inclusion of disorder in the spring model predicts four different failure regimes along the brittle-to-ductile response in agreement with experimental findings. Finally, we show that the network structure can result in stress concentration, diffuse damage, and loss of percolation depending on the failure regime. This work thus provides a framework for the design and optimization of double-network materials and underlines the importance of network structure in the toughness of polymer materials.
\end{abstract}

DOI: 10.1103/PhysRevMaterials.4.063603

\section{INTRODUCTION}

Polymer networks, such as rubbers and gels, can undergo large deformation without losing elasticity. This property makes them ideal for numerous applications, for example, in the biomedical field or in soft robotics. One of the factors that limits their applicability, however, is the brittleness of many polymer networks, which leads to inferior mechanical performance, such as low fracture toughness and strength [1]. In recent years, several strategies have been developed to toughen polymer networks by introducing dissipation mechanisms that delay crack propagation. One of the most successful strategies relies on the combination of two different polymer networks into one material, to create a so-called double network. In particular, extremely tough double-network hydrogels have been produced by interpenetrating a stiff and weak first network with a soft and extensible second network [2-11] [Fig. 1(a)]. Later, this toughening strategy was also shown to be effective for multinetwork elastomers $[12,13]$ or macroscopic composites [14,15] [Fig. 1(b)]. This suggests that the underlying principle that leads to toughening is not strongly dependent on molecular details, but governed more generally by an interplay between two mechanically different networks.

The increase in toughness of double networks is related to a transition from brittle to ductile failure. This

\footnotetext{
*justin.tauber@wur.nl

†simone.dussi@wur.nl

¥jasper.vandergucht@wur.nl
}

brittle-to-ductile transition (BDT) becomes apparent when looking at the stress-strain response of double networks subjected to tensile deformation, as schematically shown in Fig. 1(c). In contrast to single polymer networks that typically fail in a brittle fashion (blue curve), tough double networks fail in a ductile manner with significant softening and plastic deformation (leading to "necking") prior to failure (yellow curve) $[13,16]$. This macroscopic softening is believed to be due to the progressive breaking of bonds in the stiff network (which is also called the sacrificial network), while the soft matrix formed by the second network stays intact. To obtain tough materials, it is thus crucial that the rupture of the first network occurs without leading to macroscopic cracks in the material.

Several theoretical models have been proposed to explain the toughness of double networks. The first of these are due to Tanaka [17] and Brown [18] that describe failure around a defect, such as a notch or a crack. They assumed that a crack tip in the material is surrounded by a region in which the first network breaks by forming microcracks that are stabilized by bridging chains from the second network. Later extensions of these models explicitly accounted for the evolution of damage in the material and were able to describe the hysteresis in the stress-strain response, the progressive softening, and the Mullins effect [19-24]. These phenomenological models assume that the stress field in the material can be described by continuum mechanics, which treats the material as a homogeneous elastic solid. However, it is known that double networks contain large heterogeneities, which play an important role in determining their mechanics [25]. To understand how microcracks in the material nucleate 
(a)

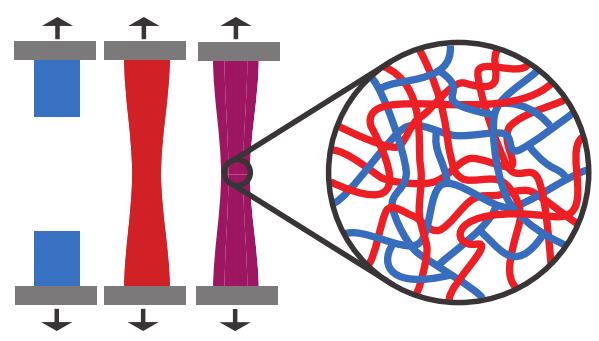

(b)
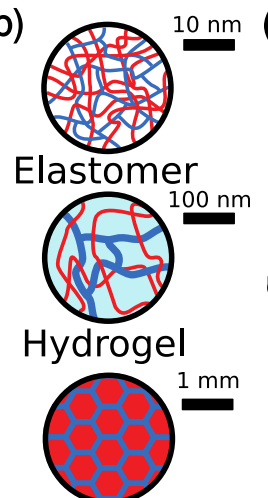

Macroscopic (c)

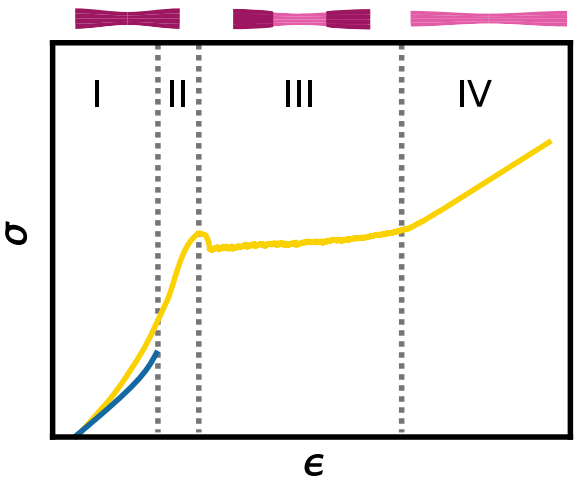

FIG. 1. Double networks across the scale. (a) By combining a sacrificial network that is stiff and weak (blue), with a matrix network that is soft and strong (red), a (molecular) composite both stiff and strong (tough) can be created. (b) Typical double networks are made of elastomers [12] and hydrogels [2], but they can also be made in the form of macroscopic composites [14]. (c) Schematic of a brittle (blue) and a ductile (yellow) stress-strain response.

and propagate and to find a criterion that predicts the onset of macroscopic failure, a model is needed that takes these heterogeneities into account.

One way to go forward is to include molecular details, for example, using molecular dynamics simulations [26-28]. However, given the computational costs it is difficult to do this for large system sizes and to explore a large range of parameters. Alternatively, statistical models, such as the fiber bundle model, can give insight into the role of disorder on network failure $[29,30]$. In such models, however, a priori assumptions must be made about the (re)distribution of stress among the different elements, so that they cannot explain the spatial evolution of damage in the materials and its relation to the structure of the networks.

Here, we develop a random double spring network model, which explicitly takes into account disorder and the resulting heterogeneous stress distributions. Rather than making assumptions about the distribution of strain and stress in the material, this distribution emerges naturally from the condition of mechanical equilibrium between the different networks. Because a network is included explicitly, the model provides information on the failure process both at the macroscopic and the microscopic levels. We demonstrate how this model predicts a simple criterion for the BDT and we show how the nature of this transition is influenced by disorder and the resulting stress localization. We also compare our network model to a simple one-dimensional (1D) multispring model, which can be solved analytically.

(a)

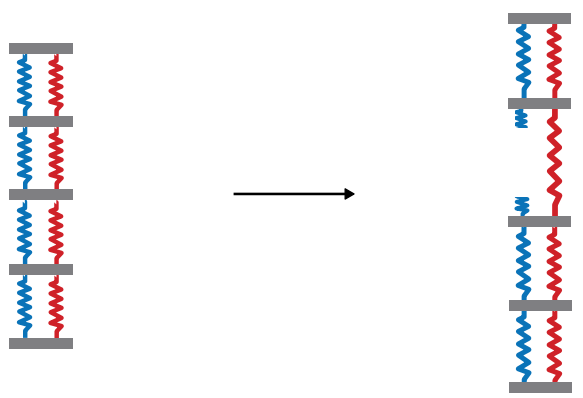

(b)
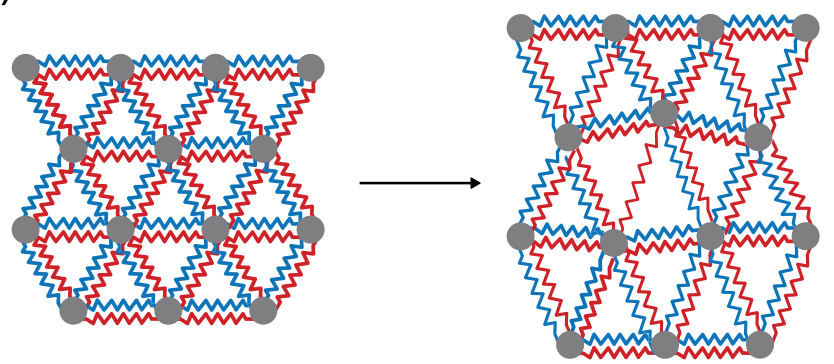

FIG. 2. Minimal models for elastic double networks and their failure response. The springs represent elements of the sacrificial network (blue) and matrix network (red). (a) Multispring (MS) model. (b) Random spring network (RSN) model. Simulations are performed for networks of $50 \times 50$ nodes.

\section{MODELS AND METHODS}

The BDT is the universal mechanical feature of doublenetwork materials. In a brittle material, a small microcrack in the sacrificial network directly leads to macroscopic failure. On the contrary, a ductile material remains intact when microcracks develop. To describe this transition, we first present a simple 1D multispring model, which is an extension of a previously used two-spring model [24,31] and which can be solved analytically [Fig. 2(a)]. While heterogeneity can be included in this 1D model, it does not take into account the network structure of the material and therefore cannot give an accurate description of the stress distribution in the material. We therefore consider a more realistic double random spring model that does allow for a heterogeneous stress distribution in the material [Fig. 2(b)].

\section{A. Multispring model}

In the multispring model (MS), the double network is modeled as a series of elements, each consisting of two parallel springs, one representing the sacrificial network (with spring constant $\mu_{S}$ ) and one representing the matrix (with spring constant $\mu_{M}$ ) [see Fig. 2(a)]. Each spring is assumed to be a linear Hookean spring, so that the force acting on it is given by $F_{i}=\mu_{i} \Delta l_{i}$, with $\Delta l_{i}$ the extension of spring $i$. Removal of a sacrificial spring in an element leads to the formation of a microcrack, which is bridged by the remaining matrix spring. As the elements are connected in series, the load on each element is equal. This force balance between the elements implies

$$
\sigma=\left(\mu_{M}+\mu_{S}\right) \Delta l_{D}=\mu_{M} \Delta l_{M},
$$


where $\Delta l_{D}$ and $\Delta l_{M}$ represent the extension of the network in the intact double network and in the matrix spanning the microcrack, respectively, and where $\sigma$ denotes the stress. The overall strain of the system $\epsilon$ can be written as

$$
\epsilon=\Delta l_{D}(1-\phi)+\Delta l_{M} \phi
$$

where $\phi$ denotes the fraction of broken sacrificial bonds (i.e., the fraction of microcracks).

Rupture of a bond is assumed to occur instantaneously when the extension of a spring $\Delta l_{i}$ exceeds its threshold $\lambda_{i}$. In the absence of disorder, all sacrificial bonds have the same threshold $\lambda_{S}$ and all matrix bonds have the same threshold $\lambda_{M}$. Brittle failure after formation of a microcrack occurs when the bridging matrix bond reaches its threshold before the other sacrificial bonds, while ductile failure occurs when the sacrificial bonds reach their threshold first. From Eq. (1) it immediately follows that the brittle-to-ductile transition occurs when $\left(\mu_{S}+\mu_{M}\right) \lambda_{S}=\mu_{M} \lambda_{M}$. We can therefore predict which system parameters are required for a system at the BDT. For example, the sacrificial bond threshold $\lambda_{S}^{*}$ for which the rupture force of a (matrix-reinforced) sacrificial bond is equal to the rupture force of a matrix bond is given by

$$
\lambda_{S}^{*}=\frac{\mu_{M} \lambda_{M}}{\mu_{S}+\mu_{M}} .
$$

More generally, we can define a parameter $\Delta \alpha$ to quantify the distance from the BDT:

$$
\Delta \alpha=\frac{\mu_{M} \lambda_{M}}{\left(\mu_{M}+\mu_{S}\right) \lambda_{S}}-1 .
$$

If $\Delta \alpha=0$, the system is at the BDT. Brittle failure occurs for $\Delta \alpha<0$; in this case, only one microcrack is enough to cause global failure so the fraction of broken sacrificial bonds at failure is $\phi_{f}=1 / N$ with $N$ the system size. Ductile failure occurs for $\Delta \alpha>0$, where all sacrificial bonds break before the system fails globally, so $\phi_{f}=1$. It follows from this analysis that for ductile failure we need $\lambda_{S}<\lambda_{M}$, which explains why the creation of tough, ductile networks requires the sacrificial network to be much weaker than the matrix.

For a perfectly homogeneous system, the BDT in this model is an abrupt transition. Experiments, however, show that the descriptors of failure vary in a continuous manner through the BDT [16]. We therefore introduce disorder by assuming that the thresholds of the sacrificial network vary according to a certain distribution $P\left(\lambda_{S}\right)$. All other parameters are taken the same. In this case, the sacrificial bonds will fail progressively, with the weakest bonds breaking first and the stronger bonds breaking later. At a given strain $\epsilon$, all sacrificial bonds for which $\lambda_{S}<\Delta l_{D}$ are broken. In the continuous limit (for $N \gg 1$ ), the fraction of broken sacrificial bonds can thus be written as

$$
\phi(\epsilon)=\int_{0}^{\Delta l_{D}} P\left(\lambda_{S}\right) d \lambda_{D} .
$$

Here, we will consider a Gaussian threshold distribution with mean $\left\langle\lambda_{S}\right\rangle$ and standard deviation $\delta \lambda$. For this case, it follows from Eqs. (1) and (5) that at the moment of macroscopic failure (where $\Delta l_{M}=\lambda_{M}$ ), the fraction of broken sacrificial bonds is given by

$$
\phi_{f}=\frac{1}{2}[1+\operatorname{erf}(\Delta \tilde{\alpha})]
$$

with

$$
\Delta \tilde{\alpha}=\frac{\Delta \alpha\left\langle\lambda_{S}\right\rangle}{\delta \lambda \sqrt{2}}
$$

a normalized parameter to quantify the distance to the BDT, which takes into account the disorder in the thresholds. The fraction of broken sacrificial bonds thus increases gradually from 0 to 1 along the BDT and the parameter $\Delta \tilde{\alpha}$ sets the steepness of this transition. With increasing disorder, the transition becomes more gradual. The strain at break follows from Eqs. (1) and (2):

$$
\epsilon_{f}=\lambda_{M}\left(\frac{\mu_{M}+\phi_{f} \mu_{S}}{\mu_{M}+\mu_{S}}\right) .
$$

In the brittle regime $\left(\Delta \tilde{\alpha} \ll-1, \phi_{f} \approx 0\right)$ we find $\epsilon_{f}=$ $\mu_{M} \lambda_{M} /\left(\mu_{M}+\mu_{S}\right)$, while in the ductile regime $(\Delta \tilde{\alpha} \gg 1$, $\phi_{f} \approx 1$ ) we have $\epsilon_{f}=\lambda_{M}$.

\section{B. Random spring network model}

Being a 1D model, the multispring model cannot account for stress heterogeneity and the resulting localization of stresses. We therefore consider a random spring network model (RSN) as shown in Fig. 2(b) composed of $L \times L$ nodes arranged on a triangular lattice whose nearest neighbors are connected by the same element we introduced for the multispring model ( $L=50$ for all RSN simulations). Now, upon applying an external load, stress concentration is possible due to the topological restrictions imposed by the network structure.

Following the experimentally found guidelines for making tough double networks [2] we consider materials in which the sacrificial network is stiff and weak, while the matrix is soft and strong. This means that the elastic constant of sacrificial springs $\mu_{S}$ is always higher than the one of the matrix springs $\mu_{M}$. We therefore vary the ratio of the stiffnesses such that $0<$ $\mu_{M} / \mu_{S}<1$. To reflect the asymmetry in network strengths, we fix $\lambda_{M}=4.0$ and vary $\lambda_{S}$ such that $\lambda_{S}<\lambda_{M}$. In all cases, we keep the parameters of all matrix springs the same. To implement disorder at the bond level, the failure thresholds $\lambda_{S}$ are picked from a Gaussian distribution as in the MS model. Nodes at the bottom and top are fixed in the $y$ direction, but they can slide along the $x$ direction. Along the $x$ axis periodic boundary conditions are implemented.

We characterize the mechanical response of the network by applying an extensional strain $\epsilon$ along the $y$ axis in small steps of $0.1 \%$ strain. We consider quasistatic loading and assume that the networks remain in mechanical equilibrium at each step, i.e., it settles in its minimum energy state. Therefore, after every step, the total energy of the network is minimized by displacing the nodes using the FIRE algorithm [32] with a tolerance of $F_{\text {rms }} \leqslant 1 \times 10^{-5}$. Here, $F_{\text {rms }}$ is the maximum root-mean-squared force allowed in the system [32]. To simulate failure, we break all bonds which exceed their failure threshold $\lambda_{i}$ consecutively. After the failure of every single bond, the energy of the system is minimized. Once all overstretched bonds are broken, the next strain step is determined according to the bond that is closest to its failure threshold with a minimum step size of $0.001 \%$ strain. 


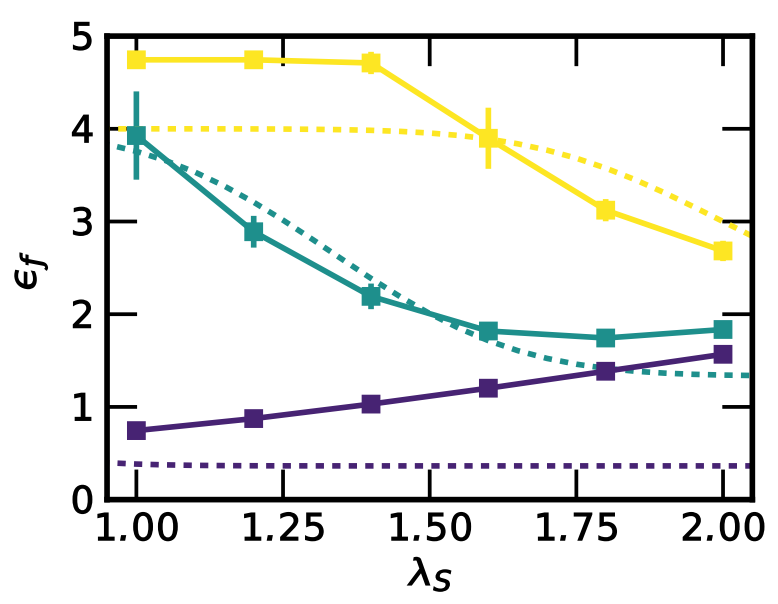

FIG. 3. Failure strain $\epsilon_{f}$ versus the average failure threshold $\left\langle\lambda_{S}\right\rangle$ in systems with $\delta \lambda=0.250$. Results are shown for the RSN model (solid lines) and the MS model (dashed lines) for stiffness ratios $\mu_{M} / \mu_{S}=0.10$ (purple), 0.50 (green), and 1.00 (yellow).

For every strain in the simulations, the virial stress is calculated from the forces exerted by the springs on the nodes. As the system is elongated along the $y$ direction, we consider the $y y$ component of the virial tensor as a measure for the stress $\sigma$. We define the softening strain $\epsilon_{\text {soft }}$ as the strain where the stiffness drops below the initial stiffness, and the failure strain $\epsilon_{f}$ and failure stress $\sigma_{f}$ as the strain and stress for which the system becomes disconnected along the $y$ direction. For every data point, 50 simulations are performed, and error bars represent standard deviation.

\section{RESULTS AND DISCUSSION}

\section{A. Failure regimes of double networks}

The aim of this paper is to systematically explore the effect of disorder and stress heterogeneity on the failure of double networks, focusing especially on the role of the network. To do this in a systematic fashion, we will extensively compare the results of the random spring network (RSN) model with the 1D multispring (MS) model lacking any network structure.

We start our comparison by checking a prediction from the MS model for the dependence of the failure strain $\epsilon_{f}$, described in Eq. (8), on the sacrificial bond properties $\lambda_{S}$ and $\mu_{M} / \mu_{S}$ in the presence of disorder (Fig. 3). Although it is clear that both the MS model and the RSN model follow the same trend, the overall trend is not immediately obvious. Intuitively, it is expected that the failure strain should increase with an increase in $\lambda_{S}$. However, only at the lowest stiffness ratio, we see that the $\epsilon_{f}$ increases with $\lambda_{S}$. At high stiffness ratios, this trend is reversed. This transition is a consequence of the brittle-to-ductile transition (BDT). From Eq. (4) it can be deduced that an increase in $\lambda_{S}$ brings the system from the ductile into the brittle regime. The transition is not observed at the lowest stiffness ratio because all those systems are already in the brittle regime. Similar trends can be found for the material strength and the work of extension (see Supplemental Material [33]).

These results show that we can tune the failure response based on the properties of the individual sacrificial bonds. (a)

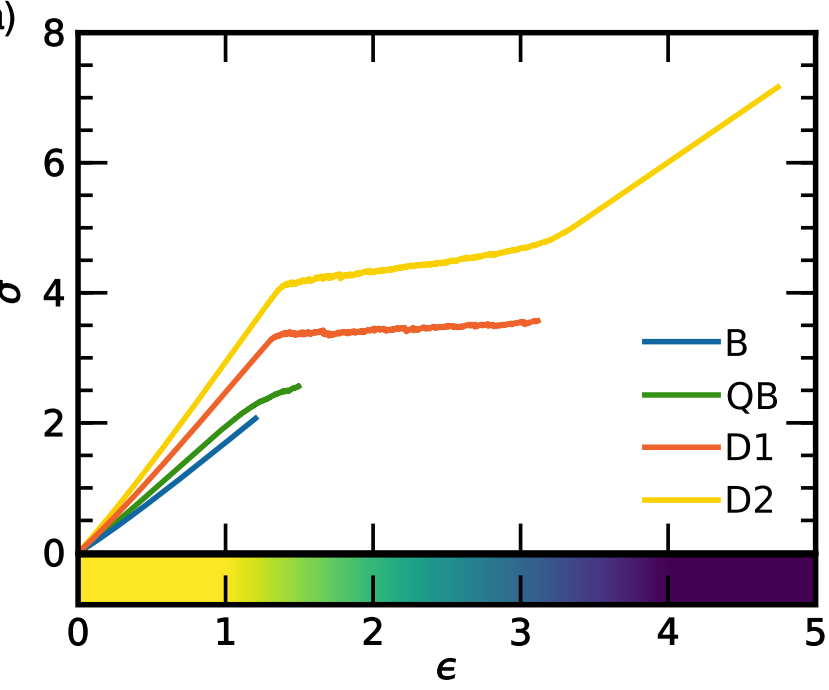

(b)

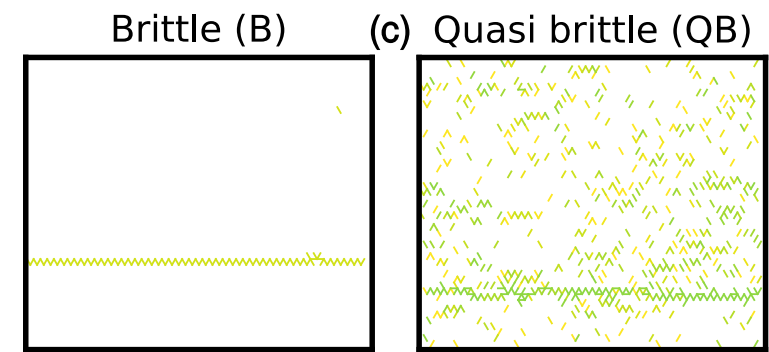

(d)
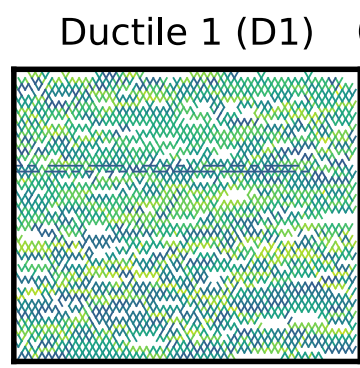

(e)

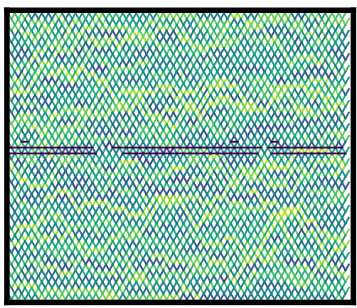

FIG. 4. Typical failure response of double networks obtained via the RSN model. (a) The four distinct stress-strain responses: brittle (B), quasibrittle (QB), the first ductile regime (D1), and the second ductile regime (D2). (b)-(e) Corresponding failure patterns. Every line represents a broken sacrificial bond, color coded according to the strain at which it failed (see color bar above).

Taking a broader view at the entire stress-strain response of the RSN, we can differentiate between four distinct responses, shown in Fig. 4(a). There are two brittle responses, brittle (B) and quasibrittle (QB), that can be distinguished by the (slight) softening that occurs before macroscopic failure in the QB case. There are also two ductile responses (D1 and D2) that both show a clear plateau in the stress-strain response after softening. In case of D1, macroscopic failure occurs within this plateau. For D2, a second increase in the stress precedes final failure. These same four responses are also found in the MS model (see Supplemental Material [33]).

By contrast, at the microscopic level the failure behavior of the RSN is distinct from the MS model. In Figs. 4(b)-4(e), we plot the broken bonds of the sacrificial network color coded according to the strain at which they break. We clearly observe that the number of broken sacrificial bonds increases from B 

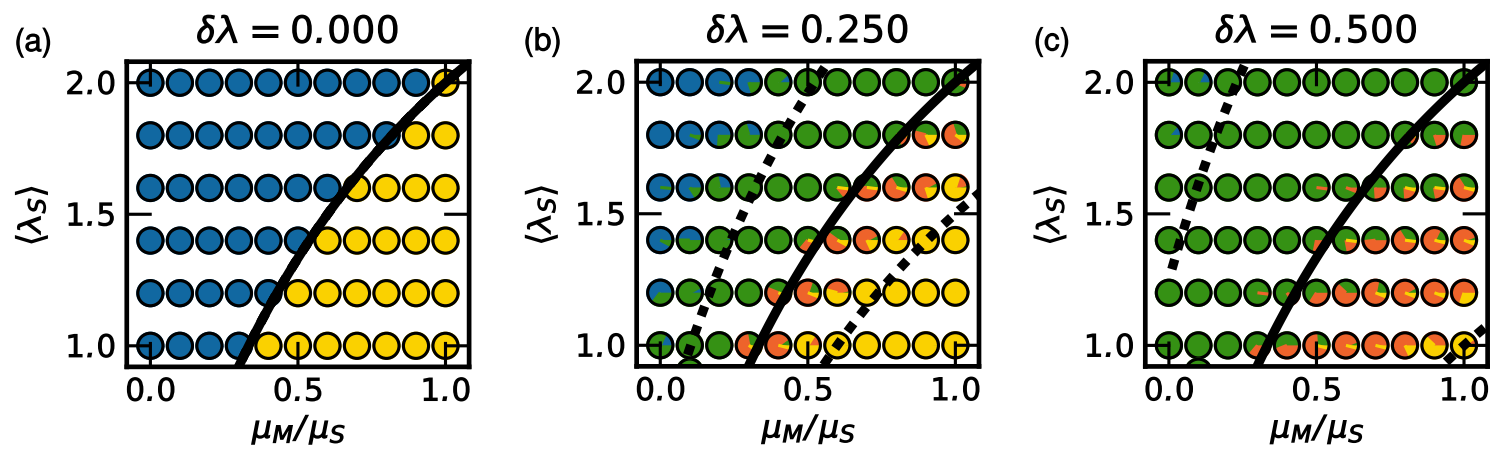

FIG. 5. Macroscopic mechanical phase diagrams in the $\left(\mu_{M} / \mu_{S}, \lambda_{S}\right)$ plane. Each panel corresponds to a different value of disorder $\delta \lambda$. Symbols are pie charts color coded according to the frequency of the four fracture regimes [B (blue), QB (green), D1 (orange), D2 (yellow)] observed from 50 independent simulations. The black line indicates the BDT according to the MS model. The dashed lines indicate the B-to-QB transition $(\Delta \tilde{\alpha}=-2.5)$ and the D1-to-D2 transition $(\Delta \tilde{\alpha}=2.0)$ in the MS model (see Supplemental Material [33]).

to D2 type of fracture. In B, all broken bonds are part of a single crack at the location where macroscopic failure occurs. A similar pattern is observed for QB, where, however, more bonds fail homogeneously throughout the sample before the final crack appears. It is the failure of these bonds that causes the softening observed in the QB stress-strain response. The ductile regimes are characterized by many bonds breaking simultaneously. Such avalanches occur over a large strain range, from the moment of softening until macroscopic failure. In case of D2 fracture, all the nonhorizontal bonds of the sacrificial network, which are the elastically active bonds, are broken. In the MS model we can not differentiate between failure patterns because the load is homogeneous throughout the system, so that stress can not concentrate and damage will always be homogeneous.

We note that both the RSN model and the MS model capture the four different mechanical responses observed in experiments on elastomers [13], hydrogels [2], and macroscopic composites $[14,15]$. The clear difference in fraction of broken sacrificial bonds between (quasi)brittle and ductile responses is also well established experimentally $[13,16]$. Furthermore, the softening response, which is irreversible both in our model and in the experiments, is attributed to early damage of bonds in the sacrificial network [22] which has been shown to occur homogeneously throughout the network well before macroscopic failure [13]. Finally, also a plateau in the stress response is typically observed in experiments after the yielding of the material, often accompanied by a necking region where only sacrificial bonds break [13]. We conclude that both the RSN and MS models represent a minimal yet insightful model to study fracture in elastic double networks both at the macroscopic and microscopic levels and, therefore, we can proceed to a more detailed analysis.

\section{B. Macroscopic characterization of the failure regimes}

The results in Fig. 3 clearly demonstrate the dependence of the failure response on the properties of the sacrificial bonds: $\left\langle\lambda_{S}\right\rangle$ and $\mu_{M} / \mu_{S}$. To study the joined effect of these properties together with the disorder in the failure threshold $(\delta \lambda)$, we construct phase diagrams of the failure response (Fig. 5) based on the four different regimes described above (see Supplemental Material [33]). For each point in the diagram we use a pie-chart symbol indicating the frequency of the four different regimes observed over 50 independent simulations. We first focus on the case with no disorder $\delta \lambda=0$, shown in Fig. 5(a), and observe that both $\mu_{M} / \mu_{S}$ and $\lambda_{S}$ control the sharp transition from the BDT. Also, the BDT of the RSN model coincides with the BDT of the MS model [Eq. (4)] as indicated by the black line.

Next, we consider the cases with disorder, plotted in Figs. 5(b) and 5(c), and observe the appearance of the intermediate regimes $\mathrm{QB}$ and D1. These two regimes appear around the BDT and upon increasing disorder $\delta \lambda$ progressively span the entire explored parameter space, which is a clear indication that disorder controls the position of these transitions. In addition, the transitions between the regimes are less sharp when the disorder is larger, as testified by the less homogeneously colored pie-chart symbols. Indeed, the mechanical responses of networks with large $\delta \lambda$ depend on the exact realization of the network (exact distribution of threshold values and their spatial organization), even when having the same $\mu_{M} / \mu_{S}$ and $\left\langle\lambda_{S}\right\rangle$, especially around boundaries between different regimes. A similar dependence of the location of the B-to-QB and D1-to-D2 transitions on disorder is also predicted by the MS model (dashed lines, see Supplemental Material for further information [33]). At low disorder, the location of the boundaries corresponds well between the models, but at high disorder the boundaries move away from each other. This comparison reveals that the type of load sharing (equal load sharing in the MS model or network controlled load sharing in the RSN model) is not essential for the occurrence of any of the four regimes. Nevertheless, the stress redistribution via the network does affect how far from the BDT these regimes occur. Furthermore, disorder clearly plays an important role also in the experimental systems since QB and D1 responses are typically observed experimentally and we show that these occur only in presence of disorder. Our results confirm that knowing the average value of the bond strengths might not be enough to predict the failure regime, in agreement with recent phenomenological models aiming to capture softening and the Mullins effect in double networks [22].

\section{Failure at the microscopic level}

In the previous section, we found that the macroscopic force balance reasonably captures the transition from 
(a)

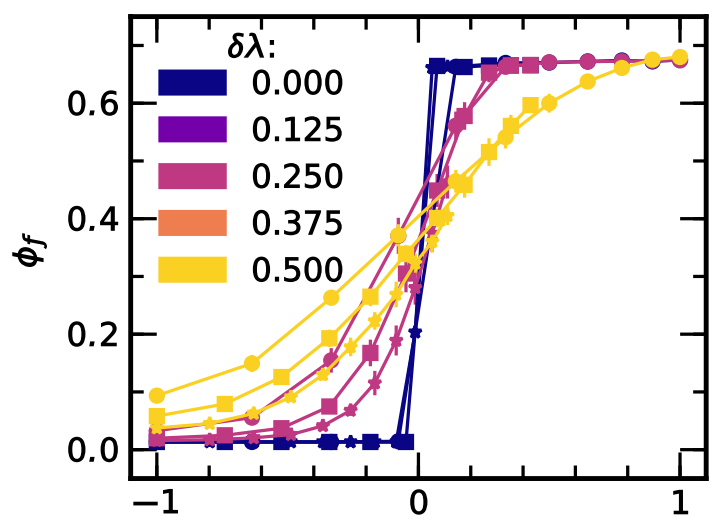

(b)

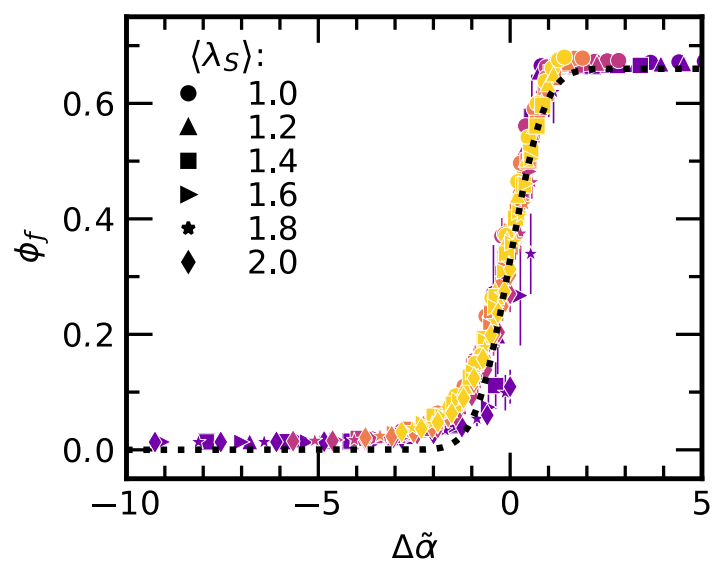

FIG. 6. Rescaling of the fraction of broken bonds $\phi_{f}$. (a) The fraction of total broken bonds $\phi_{f}$ obtained in simulations of RSN is plotted against $\Delta \alpha$, which quantifies the distance from the BDT predicted by the equal load-sharing models. (b) $\phi_{f}$ from RSN versus $\Delta \tilde{\alpha}$. The dashed black line indicates the theoretical prediction for $\phi_{f}$ according to the MS model [Eq. (4)]. In the RSN model, only the vertically aligned bonds are expected to break, therefore, the prediction of the MS model is multiplied by a factor $\frac{2}{3}$. The symbols correspond to the $\lambda_{S}$ value [see legend in (b)] color coded according to the amount of disorder $\delta \lambda$ [see legend in (a)]. Error bars indicate standard deviation based on 50 configurations.

(quasi)brittle to ductile fracture in the RSN model and that disorder influences this transition. Furthermore, we have seen in Figs. 4(b)-4(e) that as long as some disorder is present, distinct failure patterns develop in the RSN that suggest a tight link between the macroscopic and microscopic failure process. In this section, we explore several indicators of microscopic failure in disordered RSN systems, with a special focus on stress heterogeneity, and we consider to what extent the force balance influences the microscopic failure processes in double networks.

\section{Fraction of broken bonds}

The first parameter we explore is the fraction of broken sacrificial bonds at final failure $\phi_{f}$, as we have a direct prediction from the MS model. In Fig. 6(a), we plot $\phi_{f}$ as a function of $\Delta \alpha$, the distance from the BDT as introduced in Eq. (4) for various amount of disorder $\delta \lambda$. Clearly, we see a strong increase in the number of broken bonds upon going from the brittle to the ductile regime. For $\delta \lambda=0$ we observe that all data points collapse on a step function exactly at the BDT, $\Delta \alpha=0$. When disorder is introduced, the transition becomes more gradual, corresponding to the shift of the B-to-QB and D1-to-D2 transition in Fig. 5. We can understand these effects by pointing out that both weak and strong bonds are introduced due to the spread in sacrificial bond strength. For $\Delta \alpha<0, \phi_{f}$ is increased by the disorder due to the presence of weak springs that break before brittle failure occurs. Similarly, for $\Delta \alpha>0, \phi_{f}$ decreases due to disorder that introduces bonds that are too strong to break before the matrix fails.

According to the prediction for the MS model in Eq. (6) we can collapse all these data on one master curve for all values of $\lambda_{S}, \mu_{M} / \mu_{S}$, and $\delta \lambda$, if we consider the rescaled distance to the BDT: $\Delta \tilde{\alpha}$. Indeed, we obtain a good collapse for $\phi_{f}$, as shown in Fig. 6(b). However, there is a significant increase in $\phi_{f}$ in the brittle regime relative to the prediction of the MS model (dashed line) (see Supplemental Material [33]). This could be an indication that the load sharing via the network enhances the failure of sacrificial bonds, however, we can not exclude that the discrepancy is due to a finite-size effect.

\section{Stress concentration in the spring network model}

In the MS model it is assumed that stress is distributed homogeneously throughout the entire system, even after failure of sacrificial bonds. Hence, stress can never concentrate within the MS model. In the RSN model stress concentration is possible and therefore it can be used to study the nucleation and propagation of failure in double networks. It is fascinating that this fundamental difference between the MS model and the RSN model does not result in big differences in macroscopic failure or the fraction of broken sacrificial bonds.

Interestingly, a second look at the type of sacrificial bonds that break does reveal a striking difference between the MS model and the RSN model caused by the concentration of stress. By construction, in the MS model sacrificial bonds break in the order of their strength, from weak to strong, until a certain threshold $\lambda_{S}^{*}$ [Eq. (3)] is reached and macroscopic failure occurs. At this threshold $\lambda_{S}^{*}$, the force required to break a (matrix-reinforced) sacrificial bond is equal to the force required to break a matrix bond. How $\lambda_{S}^{*}$ influences the failure of bonds is further illustrated in Fig. 7(a) for a system below the BDT $\left(\Delta \tilde{\alpha}=-0.7, \mu_{M}=0.4\right.$, and $\left.\left\langle\lambda_{S}\right\rangle=1.4\right)$. For this system $\lambda_{S}^{*}=1.14$, so in the MS model only weak bonds with $\lambda_{S}<1.14$ would fail. However, in the RSN model we see that sacrificial bonds stronger than $\lambda_{S}^{*}$ also break [see Figs. 7(b) and 7(c)]. In particular, in Fig. 7(d) we plot the fraction of broken strong sacrificial bonds $\phi_{\text {strong, } f}$ (i.e., bonds with $\lambda_{S}>\lambda_{S}^{*}$ that break) as a function of the distance from the BDT $\Delta \tilde{\alpha}$ for various amounts of disorder $\delta \lambda$. At low $\delta \lambda$ and far below the BDT, failure of strong sacrificial bonds is required in order to achieve macroscopic failure since there are just not enough weak sacrificial bonds [Fig. 7(b)]. However, upon approaching the transition at $\Delta \tilde{\alpha}=0, \phi_{\text {strong, } f \text { grows, even }}$ though a decrease could be expected based on the total concentration of strong sacrificial bonds [Fig. 7(c)]. Interestingly, we observe a maximum in $\phi_{\text {strong, } f}$ before fully entering the 
(a)

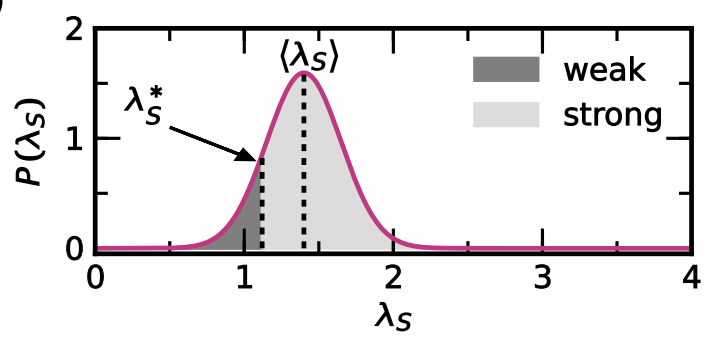

(d)

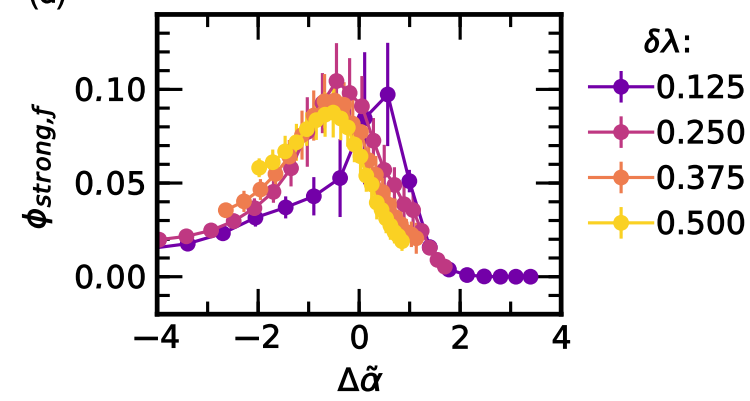

(b)

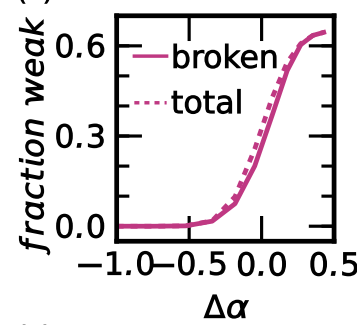

(e) 0.5

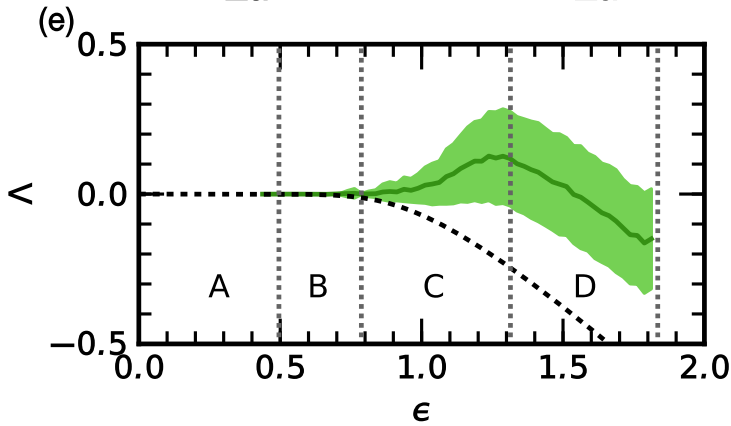

(c)

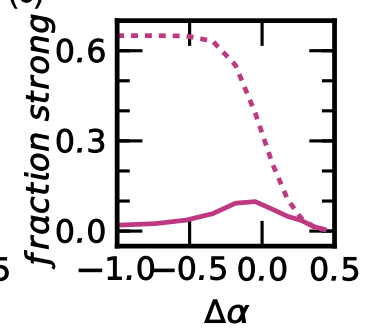

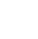

FIG. 7. Development of stress heterogeneity and stress concentration during deformation. In all subpanels, $\left\langle\lambda_{S}\right\rangle=1.4$. (a) Distribution of $\lambda_{S}$ for a system with $\left\langle\lambda_{S}\right\rangle=1.4$ and $\Delta \tilde{\alpha}=-0.7$. At $\lambda_{S}^{*}$ the force required to break a sacrificial bond is equal to the force required to break a matrix bond. Thus, if $\lambda_{S}<\lambda_{S}^{*}$ a sacrificial bond is weaker than the matrix bonds (dark gray) and if $\lambda_{S}>\lambda_{S}^{*}$ a sacrificial bond is stronger (light gray). (b) Comparison of total fraction of weak bonds that align with the deformation field and the fraction of weak bonds $\phi_{w e a k, f}$ that failed during deformation versus $\Delta \alpha$. (c) Similar comparison for the total amount of strong bonds and broken strong bonds $\phi_{\text {strong, } f}$. (d) $\phi_{\text {strong, } f}$ versus $\Delta \tilde{\alpha}$ for different $\delta \lambda$. Error bars indicate standard deviation. (e) Development of $\Lambda$, defined as the difference between the rupture threshold $\lambda_{S \text {, fail }}$ of the failing sacrificial bonds and $d l_{\text {aff }}$, the extension of the bonds if the strain field were homogeneous throughout the system, as a function of strain for a network with $\Delta \tilde{\alpha}=-0.7,\left\langle\lambda_{S}\right\rangle=1.4$, and $\delta \lambda=0.250$. The four intervals are described in the text. The average (line) and standard deviation (shaded area) are calculated from a histogram of $\lambda_{S \text {,fail }}-d l_{\text {aff }}$ over 50 simulations accumulated over bins of $2.5 \%$ width. The dashed black line indicates the prediction for $\Lambda$ according to the MS model.

ductile regime $\Delta \tilde{\alpha}>0$ where mostly weak sacrificial bonds control the fracture process. Strong bonds can only break if the force they carry exceeds the maximum force that is expected based on the macroscopic force balance. Thus, we conclude that the network structure plays a crucial role in (re)distributing the load during deformation, giving rise to stress concentration. The development of stress heterogeneity is tightly bound to the disorder that is present in the initial structure. We expect that by including more (structural) disorder in the initial network (e.g., inhomogeneities in the connectivity of the nodes, a disordered spatial distribution of the nodes, or a distribution in the stiffness of the bonds) the stress heterogeneity would become even larger [34]. As a consequence, we would expect significant broadening of the $\mathrm{QB}$ and D1 phases with respect to the prediction from the MS model.

Because in the RSN the initial structure is homogeneous, stress concentration does not occur from the onset of deformation, but develops during deformation as bonds are ruptured. We can quantify this development via the difference between the failure threshold of a broken sacrificial bond $\lambda_{S \text {,fail }}$, a measure for the actual local extension, and the extension estimated from the global strain assuming all deformations are affine, $d l_{\text {aff }}$. We therefore define $\Lambda=\lambda_{S \text {,fail }}-d l_{\text {aff }}$. To compare with the MS model, it must be noted that in this model the stress is homogeneous, but the strain field is not. In fact, the microcracks take up additional strain to compensate for the absence of the sacrificial network. This means that the deformation of the remaining sacrificial bonds is less than the affine strain, so that $\Lambda$ is always smaller than 1 in the MS model [see dashed line in Fig. 7(e)]. If at any time $\Lambda>0$, stress concentration must therefore be present.

This analysis reveals different modes of stress concentration during the failure of a RSN system as shown in Fig. 7(e). At first nothing breaks, this regime coincides with the reversibly elastic regime found at the macroscopic level (regime A). Then, sacrificial bonds start to break exactly as predicted by the affine deformation. Here, both the average stress concentration $\langle\Lambda\rangle$ and its variance are zero (regime B). Upon further increasing the deformation, the variance of $\Lambda$ starts to grow indicating stress heterogeneity in the system, quickly followed by an increase of $\langle\Lambda\rangle$ indicating stress concentration (regime $C$ ). As a result, strong sacrificial bonds also start to break and failure of weak sacrificial bonds is postponed or even prevented (see Supplemental Material [33]). Finally, we arrive at a peak in $\langle\Lambda\rangle$, after which the $\langle\Lambda\rangle$ decreases until macroscopic failure is reached (regime $D$ ). We attribute this decrease to structural relaxation of the sacrificial network via the formation of microcracks, leading to loss of rigidity and eventually loss of percolation in the sacrificial network. It must be noted that the variance in $\langle\Lambda\rangle$ remains constant, indicating that heterogeneity in stress is present until the final failure event.

\section{Crack development and stress delocalization}

The development of stress heterogeneity and stress concentration during deformation is the result of microscopic 

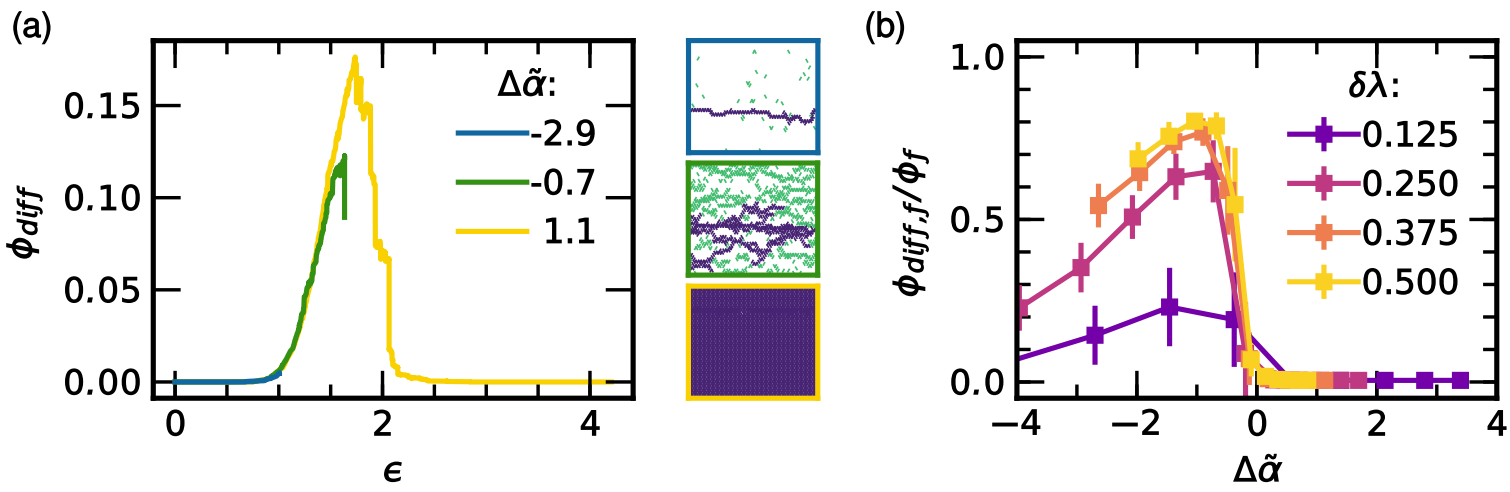

FIG. 8. Evolution of diffuse damage. (a) Fraction of broken sacrificial bonds not belonging to the largest crack $\left(\phi_{\text {diff }}\right)$ as a function of strain $\epsilon$ for different combinations of parameters below $(\Delta \tilde{\alpha}<0)$ and above $(\Delta \tilde{\alpha}>0)$ the BDT. Corresponding failure patterns in the sacrificial network are also shown, with purple bonds belonging to the largest crack and green bonds to diffuse damage. (b) Normalized diffuse damage at final failure $\phi_{\mathrm{diff}, f}$ as a function of $\Delta \tilde{\alpha}$ for different disorder $\delta \lambda$. Points are averages and error bars indicate standard deviation calculated over 50 configurations. For all curves, $\left\langle\lambda_{S}\right\rangle=1.4$.

damage evolution in the sacrificial network: the nucleation and propagation of cracks. We also suggested that the development of stress concentration is tightly linked to the disorder in the initial, undeformed, system. How crack nucleation and propagation are influenced by both initial disorder and stress heterogeneity during deformation is not trivial. We have already seen that in the RSN model, not all sacrificial bonds that break are part of the largest crack (Fig. 4). Thus, it seems that stress can be delocalized away from the crack tip of the largest crack throughout the system via diffuse damage. In this section, we quantify the evolution of (micro)cracks in the sacrificial network, focusing on diffuse damage and its coupling with stress delocalization.

In Fig. 8(a), we plot the diffuse damage, quantified by the fraction of broken sacrificial bonds (with respect to the total number of initial sacrificial bonds) that are not part of the largest crack $\phi_{\text {diff }}$ as a function of strain $\epsilon$ for three representative systems below, around, and above the BDT (see Supplemental Material [33]). In the brittle case $(\Delta \tilde{\alpha}=$ $-2.9), \phi_{\text {diff }}$ barely increases and fracture occurs at smaller strain. Closer to the BDT $(\Delta \tilde{\alpha}=-0.7)$, the diffuse damage significantly increases, reaches a maximum, and just before final failure slightly decreases. This decrease in the curve indicates that microcracks merge into the largest crack that eventually breaks the system [Fig. 8(a)]. Beyond the BDT $(\Delta \tilde{\alpha}=1.1)$, the diffuse damage reaches a higher maximum of more than $15 \%$ of the total amount of sacrificial bonds before a significant decrease that extends for a large strain interval. Finally, $\phi_{\text {diff }}$ reaches zero, indicating that all microcracks are now merged in a single large crack or damage zone, well before the final failure and all subsequent failing sacrificial bonds join this large crack.

With respect to the global stress-strain response, diffuse failure is expected to be most influential before the BDT. In the ductile regime, broken bonds will eventually join to form one large damage zone. However, in the brittle regime, many bonds can break in a diffuse fashion without being part of the largest crack, potentially diminishing stress concentration. We therefore focus on the final ratio of the broken sacrificial bonds belonging to the diffuse damage $\phi_{\text {diff, } f}$ as an indicator of stress delocalization and plot it versus $\Delta \tilde{\alpha}$ for various amounts of disorder in Fig. 8(b). We observe a nonmonotonic behavior with a peak slightly before the BDT. At the BDT, $\phi_{\text {diff, } f}$ drops drastically as all bonds eventually join the largest crack. We conclude that just before the BDT, the diffuse damage is maximum and therefore hypothesize that the crack nucleation is delayed the most in this region. A consequence of this delayed nucleation is the widening and branching of the largest crack as shown in Fig. 8(a) (snapshot in the middle). The widening of the damage zone in the sacrificial network around a defect is often used as an explanation for the enhanced fracture toughness in double-network materials $[2,18]$. Although our simulations do not measure the fracture toughness directly, our results suggest that widening of the damage zone and therefore fracture toughness is maximal just before the BDT.

\section{Relating microscopic events to the macroscopic failure regimes}

The network and the heterogeneous stress distribution emerging upon deformation clearly have a huge impact on the microscopic failure mechanism. We have seen that which microscopic failure mechanisms occur is controlled by the distance from the BDT. In addition, we have observed that these failure mechanisms can be connected to failure events that occur as a function of the applied strain. Here, we will unify our microscopic insights with the macroscopic failure regimes described earlier based on the strain values at which these events occur. These results are summarized in Fig. 9.

At the macroscopic level we identify two characteristic strains: the softening strain $\epsilon_{\mathrm{soft}}$, where the stiffness $(d \sigma / d \epsilon)$ drops below the initial stiffness [see Fig. 4(a)], and the failure strain $\epsilon_{f}$ where the system breaks in two pieces. At the microscopic level we identify strain points of three distinct events: the strain $\epsilon_{\Lambda \text {,max }}$ corresponding to the maximum in stress concentration [peak in Fig. 7(e)], the strain where the diffuse damage is maximal $\epsilon_{\text {diff,max }}$, corresponding to the peak in Fig. 8(a), and finally the strain where the (geometrical) percolation of the network of sacrificial bonds is lost $\epsilon_{\text {perc }}$ (i.e., 


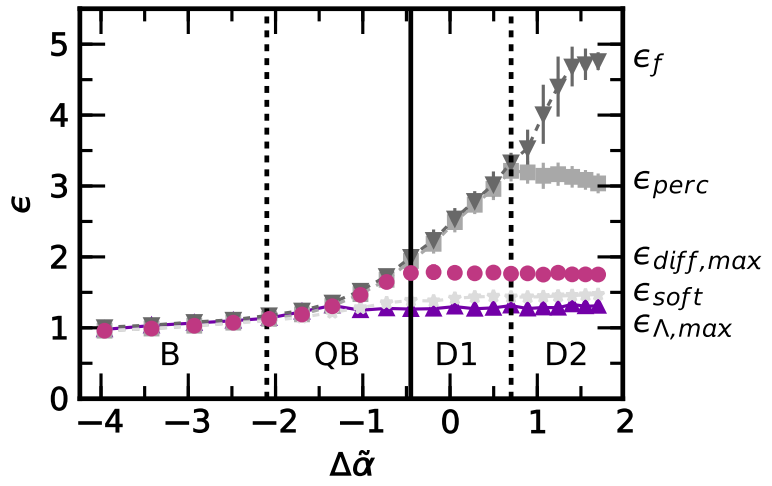

FIG. 9. Characteristic strains corresponding to microscopic and macroscopic events as a function of $\Delta \tilde{\alpha}\left(\left\langle\lambda_{S}\right\rangle=1.4\right.$ and $\delta \lambda=$ $0.250) . \epsilon_{\Lambda, \max }$ (purple triangles) is the strain at maximum stress concentration [see Fig. 7(e)]. $\epsilon_{\text {diff,max }}$ (pink circles) is the strain at which the diffuse damage is maximal [see Fig. 8(a)]. $\epsilon_{\text {perc }}$ (gray squares) is the strain at which percolation in the sacrificial network is lost. $\epsilon_{\text {soft }}$ (light gray downward triangles) indicates the onset of softening in the stress-strain curve [see Fig. 4(a) and Supplemental Material [33]]. $\epsilon_{f}$ (dark gray stars) corresponds to final fracture. The vertical lines indicate the transitions between the failure regimes in the RSN estimated from the phase diagram in Fig. 5(b).

the top and bottom of the sacrificial network are no longer connected).

$B$ regime. In the brittle regime, far below the $\mathrm{BDT}$, the system deforms homogeneously and every microcrack immediately develops into a macroscopic crack, leading to global failure. Therefore, all failure events, both macroscopically and microscopically, occur at the failure strain $\epsilon_{f}$.

$Q B$ regime. Approaching the BDT from below, we arrive at the $\mathrm{QB}$ regime, where global failure is preceded by softening in the stress-strain curve. The onset of this softening behavior $\epsilon_{\text {soft }}$ becomes distinguishable from the failure strain $\epsilon_{f}$ around the transition from B-to-QB. Interestingly, the softening strain does not depend strongly on the distance from the BDT, $\Delta \tilde{\alpha}$ (Fig. 9). If we direct our attention to the microscopic level, we see that the onset of softening coincides with the peak in stress concentration $\Lambda$ [Fig. 7(e)]. Also, for $\epsilon_{\Lambda \text {, max }}$ we only see a weak dependence on the distance from the BDT (Fig. 9). Here, we would like to stress that even though the softening strain is the first clear macroscopic sign of failure, it is not the strain at which the first bonds break. Actually, a significant amount of (weak) bonds break homogeneously throughout the system before the softening strain. However, the creation of these microcracks does not have a big effect on the stress-strain response because the bonds surrounding these microcracks absorb the released stress, leading to the increase in stress concentration in Fig. 7(e). At the softening strain, such a large amount of microcracks has been formed that the system can release (a part of) the stress caused by an applied strain via structural relaxation, i.e., the rearrangement of nodes and springs, causing the softening in the stress-strain curve in Fig. 4(a) and the drop in $\Lambda$ in Fig. 7(e). Also after $\epsilon_{\text {soft }}$, we mostly observe the creation of new microcracks or a minor expansion of existing microcracks as indicated by the strong rise of diffuse failure (Fig. 8). However, in the QB regime, just before final failure, most of these microcracks merge into one large macroscopic crack, leading to global failure as is visible in the middle snapshot in Fig. 8.

D1 regime. After the $\mathrm{BDT}$, the merging of microcracks starts to occur well before system failure, as can be identified from a peak at $\epsilon_{\text {diff,max }}$ in the diffuse failure as a function of strain [see Fig. 8(a)]. In the ductile regime, the merging of cracks occurs at the same strain, as indicated by the fact that $\epsilon_{\text {diff,max }}$ is independent of the distance from the BDT. Even though microscopic cracks in the sacrificial network start to merge, this does not mean that the sacrificial network breaks completely, i.e., loses (geometrical) percolation. In fact, in the D1 regime for a system with sufficient disorder in $\lambda_{S}$, loss of percolation in the sacrificial network only occurs around the failure strain (Fig. 9). Thus, in a system with a network structure such as the RSN, the criterion for entering the plateau region in the stress-strain curve (Fig. 4) does not have to be loss of percolation in the sacrificial network, but rather the opportunity for structural relaxation within the sacrificial network. This observation provides an alternative mechanism with respect to what was proposed in literature, where loss of percolation was thought to be necessary to enter the ductile or necking regime [9,35].

D2 regime. In the $\mathrm{D} 2$ regime, the stress-strain curve enters a matrix dominated regime after the plateau in stress [see Fig. 4(a)]. Microscopically, this transition is marked by the loss of percolation in the sacrificial network. From the loss of percolation onward, the stress in the material is carried by the matrix as is also apparent from the constant slope in the stress-strain curve [Fig. 4(a)]. However, we also observe that $\epsilon_{f}$ continues to grow with increasing $\Delta \tilde{\alpha}$, until a plateau is reached in the failure strain as we have observed earlier in Fig. 3. The initial increase of $\epsilon_{f}$ in the D2 regime shows that although percolation is lost in the sacrificial network and the matrix carries most of the stress, the sacrificial network can still contribute to the mechanical properties. Finally, we emphasize that during all these processes the matrix network remains largely intact and only very close to macroscopic failure the matrix bonds break (see Supplemental Material [33]).

\section{E. Connecting the model to experiments}

In our model, we explored how stiffness $\left(\mu_{M}\right.$ and $\left.\mu_{S}\right)$ and extensibility (strain at break of a single spring $\lambda_{M}$ and $\lambda_{S}$ ) of the individual networks influence the failure behavior of double networks. Typically, in an experimental system, such as a hydrogel or an elastomer, those parameters cannot be tuned independently. For example, the stiffness is set by the density of polymer chains [36] and the extensibility is set by the length of these chains [37]. As a first approximation, the volume fraction of monomers determines the density of polymer chains, and thus the stiffness $(\mu)$, whereas the ratio between the volume fraction of monomer and crosslinker determines the chain length, and thus the extensibility $(\lambda)$. This is only an approximation because at high monomer volume fractions, both intranetwork and internetwork entanglements will occur. These entanglements can act as effective crosslinks, increasing the effective chain density and reducing the effective chain length [38,39]. 
Nevertheless, within this approximate mapping, we find that experimental results for hydrogel and elastomer double networks are qualitatively consistent with the predictions of our model. For example, hydrogels show a transition from a brittle to a ductile failure response if the volume fraction of the matrix monomer is increased (i.e., an increase in $\mu_{M}$ in the model, consistent with Fig. 5) [4,31]. Moreover, experiments on hydrogels and elastomers reveal that an effective way to make a tough double network is to swell the sacrificial network $[3,13,16,31]$, which prestretches the polymer chains in the sacrificial network. The prestretching reduces the extensibility of the sacrificial network, which can be captured in our model by decreasing $\lambda_{s}$. In agreement with experiments, we observe in Fig. 5 that by reducing $\lambda_{S}$ we can enter the ductile failure regime. If the matrix monomers are used to swell the network, both $\lambda_{S}$ and $\mu_{M} / \mu_{S}$ are affected, revealing that the experimental swelling protocol ultimately determines which regions of the phase diagrams in Fig. 5 can be explored. Furthermore, our model shows that disorder tunes the macroscopic failure response away from the BDT, such as the B-toQB transition and the D1-to-D2 transition. In experiments, the introduction of voids can be one method to introduce disorder in a controlled way. For example, in Ref. [40] a shift in the failure response is observed from D2 to D1 with an increase in void size. In the context of our model, this corresponds to an increase in disorder and can be explained as a decrease in $\Delta \tilde{\alpha}$ [Eq. (7)].

Finally, recent advances in mechanochemistry made it possible to explore the failure response at the microscopic level as well, revealing significant diffuse damage, even before the yielding point (Fig. 7) [12,13]. In addition, scattering experiments show evidence of the delocalization of stress by probing the length scale of the nonaffine response [41] and the expansion of microscopic defects [42]. In particular, Ref. [42] suggests that a higher fraction of matrix monomer leads to the creation of new microcracks instead of the expansion of existing defects (Fig. 8). These observations fit within our theoretical framework where the distance from the BDT and disorder provide control over the microscopic failure mechanism.

The current simulation framework can be extended to increase the accuracy of its predictions for polymeric systems such as hydrogels and elastomers. In particular, we expect that the implementation of nonlinear springs will better represent the nonlinear stress-strain response of finitely extensible polymers and the redistribution of stress in a polymer network. With such an extended model we could explore if nonlinear elasticity enhances stress delocalization with respect to linear elasticity and if this effect influences nucleation and propagation of microcracks.

\section{SUMMARY AND CONCLUSIONS}

In this paper we studied both the macroscopic and microscopic failure behavior of double-network materials using a spring network model (RSN) with emergent load sharing. By comparing the results of the RSN with a model based on equal load sharing, we reveal the following: (i) The location of the BDT, defined on the basis of macroscopic stress-strain behavior, is captured by a simple force balance. (ii) Disorder introduces intermediate failure regimes but it can be incorporated in the parameter $\Delta \tilde{\alpha}$ to correctly describe the distance from the BDT, allowing for rescaling of the number of total broken sacrificial bonds. (iii) At the microscopic level, stress concentration and delocalization reveal a markedly different picture compared to global load sharing.

The overall picture that emerges from the RSN is that the force balance, a central feature of double networks, has significant control over both the macroscopic and microscopic failure behavior, irrespective of how stress is (re)distributed. By contrast, the nucleation and propagation of (micro)cracks is also highly dependent on the mode of stress (re)distribution. In particular, we have identified how stress concentration, diffuse damage, and loss of percolation are related to the transitions from B-to-QB, QB-to-D1, and D1-to-D2, respectively.

We highlight that, because many microcracks can form before global failure due to the stabilization by the matrix, the load sharing in double networks becomes highly nonlinear as a result of the interaction between these microcracks. Therefore, double networks provide a unique opportunity to exploit these nonlinearities in microcrack interaction. For example, this knowledge about the microscopic failure process could aid the development of robust self-healing double networks, as the healing of diffuse damage is easier than the healing of macroscopic cracks $[11,43,44]$.

By extending the RSN model, additional features of double-network gel failure could be studied, such as the influence of prestress or structural disorder in the sacrificial network. Furthermore, the introduction of disorder in the matrix would allow to also study the enhancement of strength and toughness, including the role of microscopic failure. In conclusion, this paper demonstrates that a random spring network model provides the opportunity to systematically study the microscopic failure process within double networks.

\section{ACKNOWLEDGMENTS}

The work of J.T., S.D., and J.v.d.G. is part of the SOFTBREAK project funded by the European Research Council (ERC Consolidator Grant No. 682782).
[1] C. Creton, 50th anniversary perspective: Networks and gels: Soft but dynamic and tough, Macromolecules 50, 8297 (2017).

[2] J. P. Gong, Why are double network hydrogels so tough? Soft Matter 6, 2583 (2010).

[3] T. Nakajima, Y. Fukuda, T. Kurokawa, T. Sakai, U. I. Chung, and J. P. Gong, Synthesis and fracture process analysis of double network hydrogels with a well-defined first network, ACS Macro Lett. 2, 518 (2013).

[4] J. P. Gong, Y. Katsuyama, T. Kurokawa, and Y. Osada, Doublenetwork hydrogels with extremely high mechanical strength, Adv. Mater. 15, 1155 (2003).

[5] L. Weng, A. Gouldstone, Y. Wu, and W. Chen, Mechanically strong double network photocrosslinked hydrogels from 
N,N-dimethylacrylamide and glycidyl methacrylated hyaluronan, Biomaterials 29, 2153 (2008).

[6] J. Y. Sun, X. Zhao, W. R. Illeperuma, O. Chaudhuri, K. H. Oh, D. J. Mooney, J. J. Vlassak, and Z. Suo, Highly stretchable and tough hydrogels, Nature (London) 489, 133 (2012).

[7] T. Nakajima, H. Sato, Y. Zhao, S. Kawahara, T. Kurokawa, K. Sugahara, and J. P. Gong, A universal molecular stent method to toughen any hydrogels based on double network concept, Adv. Funct. Mater. 22, 4426 (2012).

[8] H. Xin, S. Z. Saricilar, H. R. Brown, P. G. Whitten, and G. M. Spinks, Effect of first network topology on the toughness of double network hydrogels, Macromolecules 46, 6613 (2013).

[9] T. Nakajima, T. Kurokawa, S. Ahmed, W. L. Wu, and J. P. Gong, Characterization of internal fracture process of double network hydrogels under uniaxial elongation, Soft Matter 9, 1955 (2013).

[10] Q. Chen, L. Zhu, L. Huang, H. Chen, K. Xu, Y. Tan, P. Wang, and J. Zheng, Fracture of the physically cross-linked first network in hybrid double network hydrogels, Macromolecules 47, 2140 (2014).

[11] X. Yan, Q. Chen, L. Zhu, H. Chen, D. Wei, F. Chen, Z. Tang, J. Yang, and J. Zheng, High strength and self-healable gelatin/polyacrylamide double network hydrogels, J. Mater. Chem. B 5, 7683 (2017).

[12] E. Ducrot, Y. Chen, M. Bulters, R. P. Sijbesma, and C. Creton, Toughening elastomers with sacrificial bonds and watching them break, Science 344, 186 (2014).

[13] P. Millereau, E. Ducrot, J. M. Clough, M. E. Wiseman, H. R. Brown, R. P. Sijbesma, and C. Creton, Mechanics of elastomeric molecular composites, Proc. Natl. Acad. Sci. USA 115, 9110 (2018).

[14] R. Takahashi, T. L. Sun, Y. Saruwatari, T. Kurokawa, D. R. King, and J. P. Gong, Creating stiff, tough, and functional hydrogel composites with low-melting-point alloys, Adv. Mater. 30, 1 (2018).

[15] D. R. King, T. Okumura, R. Takahashi, T. Kurokawa, and J. P. Gong, Macroscale double networks: design criteria for optimizing strength and toughness, ACS Appl. Mater. Inter. 11, 35343 (2019).

[16] T. Matsuda, T. Nakajima, Y. Fukuda, W. Hong, T. Sakai, T. Kurokawa, U. I. Chung, and J. P. Gong, Yielding criteria of double network hydrogels, Macromolecules 49, 1865 (2016).

[17] Y. Tanaka, A local damage model for anomalous high toughness of double-network gels, Europhys. Lett. 78, 56005 (2007).

[18] H. R. Brown, A model of the fracture of double network gels, Macromolecules 40, 3815 (2007).

[19] X. Wang and W. Hong, Pseudo-elasticity of a double network gel, Soft Matter 7, 8576 (2011).

[20] X. Zhao, A theory for large deformation and damage of interpenetrating polymer networks, J. Mech. Phys. Solids 60, 319 (2012).

[21] M. Bacca, C. Creton, and R. M. McMeeking, A model for the Mullins effect in multinetwork elastomers, J. Appl. Mech. 84, 121009 (2017).

[22] F. J. Vernerey, R. Brighenti, R. Long, and T. Shen, Statistical damage mechanics of polymer networks, Macromolecules 51, 6609 (2018).

[23] S. R. Lavoie, P. Millereau, C. Creton, R. Long, and T. Tang, A continuum model for progressive damage in tough multinetwork elastomers, J. Mech. Phys. Solids 125, 523 (2019).
[24] K. Okumura, Toughness of double elastic networks, Europhys. Lett. 67, 470 (2004).

[25] Y. Kawauchi, Y. Tanaka, H. Furukawa, T. Kurokawa, T. Nakajima, Y. Osada, and J. Ping Gong, Brittle, ductile, pastelike behaviors and distinct necking of double network gels with enhanced heterogeneity, J. Phys. Conf. Ser. 184, 012016 (2009).

[26] S. S. Jang, W. A. Goddard, M. Yashar, and S. Kalani, Mechanical and transport properties of the poly(ethylene oxide)poly (acrylic acid) double network hydrogel from molecular dynamic simulations, J. Phys. Chem. B 111, 1729 (2007).

[27] W. Wang, Z. Zhang, T. Davris, J. Liu, Y. Gao, L. Zhang, and A. V. Lyulin, Simulational insights into the mechanical response of prestretched double network filled elastomers, Soft Matter 13, 8597 (2017).

[28] Y. Higuchi, K. Saito, T. Sakai, J. P. Gong, and M. Kubo, Fracture process of double-network gels by coarse-grained molecular dynamics simulation, Macromolecules 51, 3075 (2018).

[29] K. Kovács, R. C. Hidalgo, I. Pagonabarraga, and F. Kun, Brittleto-ductile transition in a fiber bundle with strong heterogeneity, Phys. Rev. E 87, 042816 (2013).

[30] S. Roy, S. Biswas, and P. Ray, Modes of failure in disordered solids, Phys. Rev. E 96, 063003 (2017).

[31] S. Ahmed, T. Nakajima, T. Kurokawa, M. Anamul Haque, and J. P. Gong, Brittle-ductile transition of double network hydrogels: Mechanical balance of two networks as the key factor, Polymer 55, 914 (2014).

[32] E. Bitzek, P. Koskinen, F. Gahler, M. Moseler, and P. Gumbsch, Structural Relaxation Made Simple, Phys. Rev. Lett. 97, 170201 (2006).

[33] See Supplemental Material at http://link.aps.org/supplemental/ 10.1103/PhysRevMaterials.4.063603 for details on analysis and additional results.

[34] S. Dussi, J. Tauber, and J. van der Gucht, Athermal Fracture of Elastic Networks: How Rigidity Challenges the Unavoidable Size-Induced Brittleness, Phys. Rev. Lett. 124, 018002 (2020).

[35] Y. H. Na, Y. Tanaka, Y. Kawauchi, H. Furukawa, T. Sumiyoshi, J. P. Gong, and Y. Osada, Necking phenomenon of doublenetwork gels, Macromolecules 39, 4641 (2006).

[36] H. M. James and E. Guth, Theory of the elastic properties of rubber, J. Chem. Phys. 11, 455 (1943).

[37] G. J. Lake and A. G. Thomas, The strength of highly elastic materials, Proc. R. Soc. A 300, 108 (1967).

[38] N. R. Langley and K. E. Polmanteer, Relation of elastic modulus to crosslink and entanglement concentrations in rubber networks, J. Polym. Sci. Polym. Phys. Ed. 12, 1023 (1974).

[39] H. Tsukeshiba, M. Huang, Y. H. Na, T. Kurokawa, R. Kuwabara, Y. Tanaka, H. Furukawa, Y. Osada, and J. P. Gong, Effect of polymer entanglement on the toughening of double network hydrogels, J. Phys. Chem. B 109, 16304 (2005).

[40] T. Nakajima, H. Furukawa, Y. Tanaka, T. Kurokawa, and J. P. Gong, Effect of void structure on the toughness of double network hydrogels, J. Polym. Sci., Part B: Polym. Phys. 49, 1246 (2011).

[41] E. Ducrot, H. Montes, and C. Creton, Structure of tough multiple network elastomers by small angle neutron scattering, Macromolecules 48, 7945 (2015).

[42] K. Fukao, T. Nakajima, T. Nonoyama, T. Kurokawa, T. Kawai, and J. P. Gong, Effect of relative strength of two networks on 
the internal fracture process of double network hydrogels as revealed by in situ small-angle $\mathrm{x}$-ray scattering, Macromolecules 53, 1154 (2020).

[43] H. Jia, Z. Huang, Z. Fei, P. J. Dyson, Z. Zheng, and X. Wang, Unconventional tough double-network hydrogels with rapid mechanical recovery, self-healing, and self-gluing properties, ACS Appl. Mater. Inter. 8, 31339 (2016).

[44] S. Liu and L. Li, Ultrastretchable and self-healing doublenetwork hydrogel for $3 \mathrm{~d}$ printing and strain sensor, ACS Appl. Mater. Inter. 9, 26429 (2017). 